\title{
Design and Simulation Analysis of Linear Loading System Based on Axial-Magnetized Voice-Coil Motor
}

\author{
Zhongliang Zhang ${ }^{1, *}$ and Liang Yan ${ }^{2}$ \\ ${ }^{1}$ Sino-French Engineering School Beihang University, Beijing, China 100191 \\ ${ }^{2}$ School of Automation Science and Electrical Engineering Beihang University, Beijing, China 100191 \\ ${ }^{*}$ Corresponding author
}

\begin{abstract}
As an important branch of linear motors, linear oscillating motor with high frequency provides reciprocating motion within short-stroke, which shows high efficiency, large power density and convenient maintenance, and has an important application value in electric vehicles, aviation and medical field. However, recent researches mostly focus on the structure design of linear motors and control strategies, rarely on its loading system. Traditional loading methods such as magnetic powder brake and weight loading cannot satisfy its special motion pattern and high frequency response requirement. For solving this problem, this paper designs an axial-magnetized voice-coil motor as the force loading system, effectively realizing various types of loading force simulation, such as spring, viscosity and hydraulic square wave force, which provides an effective solution and experimental means for the comprehensive analysis of linear oscillating motor performance.
\end{abstract}

Keywords-linear oscillating motor; force loading system; axial magnet array; voice-coil motor

\section{INTRODUCTION}

Linear motor is a kind of machine that transforms electric power directly into kinetic power based on electromagnetic principle, without any motion conversion mechanisms. Due to the advantages of high efficiency, long lifespan and low abrasion, linear oscillating motor is widely applied in electric hammers, linear pumps, compressors and other industrial applications[1,2]. Actually, advanced progress of power electronics and demand of clear energy promote the development of electric vehicle industry, in which linear motors play an important role. Wang proposes an active suspension system, which is suitable for both conventional and novel electric vehicles[3,4]. Moreover, linear motor is also applied in free piston engine[5]. Above all, improving output performance of linear motors depends on both structure design optimization and on output evaluation system.

To the present, the existing researches are mainly about optimized magnetic circuit design, efficiency analysis and motion control strategy. Zhu's study proposes multiple types of magnet arrays and conducts detailed modeling analysis for further structure optimization enhance motor's force density[6,7]. As for linear motor control, various control strategies on accurate position tracking are discussed[8,9]. Wang fulfilled the detent force compensation control, but no external load force is involved[10]. Hwang applied the compensation of the ripple force obtained by the Jacobian linearization observer to improve the position control accuracy. While the ripple force is relatively small, effects of the external force load on the motor are not given[11]. However, all these researches above concentrate on the motor itself and its dynamic performance and working state especially with loading, are not taken into account. Moreover, in some special applications, such as compressors, the external force exerted on linear motors is complicated and difficult to simulate. Thus, it's necessary to design suitable loading system to comprehensively analyze its output system.

As for loading system, there are rare researches specially for linear oscillating motor with high frequency and the uniform standard is not formed. Generally, the loading types can be classified into three categories, i.e., mechanical force loading, compressed air or hydraulic linear loading and loading with another motor. For the first load type, in spite of simple design, unidirectional load for weight loading and non-linear features for friction loading are the limitations as the force loading system for linear oscillating motors[12]. Though compressed load is utilized in working state test of compressors[13], its directional features and serve elasticity complicates the test process. The hydraulic method has the pros of large load force[14], however it cannot satisfy high frequency response requirement of linear oscillating motor. For loading with another motor, magnetic powder brake is a tradition and mature instrument for rotary motor load simulation, but not suitable for linear motor because of rotary damping on the shaft. Rotary motor with ball screw is utilized in some studies for linear motor loading. Although the thrust perturbation of linear motor has little influence on loading motor, it isn't suitable for the case in which the loading force changes frequently. After listing the advantages and the disadvantages of existing force loading methods above, for linear oscillating motor with high frequency, stable and bidirectional loading force and fast response are the most important factors. Taking all things into consideration, motorto-motor is the best method to achieve fast response linear loading. Comparing with other types of linear motor and magnet array, voice-coil motor is chosen because of its low inertia and stable output thrust within stroke. 
In this paper, a voice-coil linear motor based on axialmagnetized array is designed for achieving constant output thrust, fast response and low eddy loss in loading simulation. As the structure of force loading system, the coil mover, which is made of polyetheretherketone (PEEK) in order to control weight and avoid eddy current effect, is directed connected to linear oscillating motor shaft and placed in permanent magnetic field generated by three axial-magnetic segments. Based on this design, the magnetic field, thrust and thrust stability of voicecoil motor is simulated and analyzed in details.

The rest contents are organized as follows. The structures of voice-coil motor and loading system are introduced in section II. Magnetic field and thrust modeling with equivalent magnetic circuit method are conducted in section III. After that, section IV conducts FEM analysis and parameters optimization. At last, section $\mathrm{V}$ gives the conclusion.

\section{STRUCTURE OF LOADING MOTOR AND SySTEM}

\section{A. Voice-Coil Loading Motor}

In general, the voice-coil loading motor constitutes of two parts: the stator and the mover. Three groups of magnets with the same magnetization direction are separated by two iron rings, forming the stator. Axial-magnetized array type is selected due to the features of large length to diameter ratio of working space. Besides, the mover, made up of coppers windings and winding supporting frame, is placed in the air gap between the stator and outer back-iron of motor. Due to the radial component of permanent magnetic field generated by magnets, the mover provides axial thrust when copper windings are energized. The end cover and the outer back-iron build the flux conducting paths in order to reduce magnetic leaks and take full advantage of permanent magnetic field. The structure of motor and the flux lines of magnetic circuit are illustrated in Fig. I for better understanding. Permanent magnets are regards as magnetic sources which generate magnetic flux into inner irons. Then the magnetic flux passes the air gap and coil windings, enters into the outer back-iron, flows through the end cover and finally returns back to the other pole of magnets to realize a complete magnetic circuit.

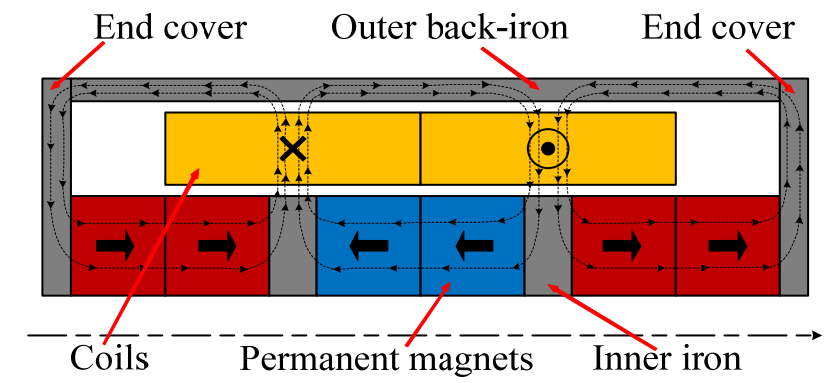

\section{FIGURE I. STRUCTURE AND MAGNETIC CIRCUIT FLUX LINES OF VOICE-COIL MOTOR}

Moreover, the mechanical structure of voice-coil motor is important for supporting and fixing as shown in Fig. II. The output shaft is made of polyetheretherketone (PEEK), which is non-conductive and non-ferromagnetic, in order to reduce weight and eliminate the eddy current effect simultaneously. In addition, the non-ferromagnetic core tube, made of stainless steel, is utilized to fix magnets and iron rings and they are locked tightly by screws on end covers of motor.

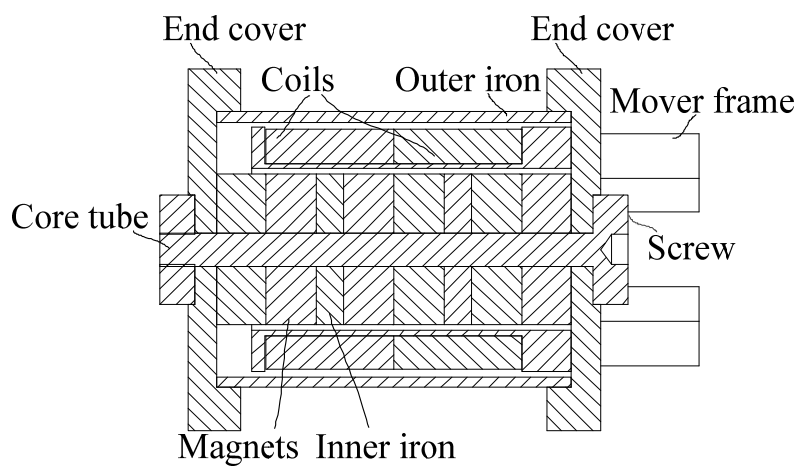

FIGURE II. MECHANICAL STRUCTURE OF VOICE-COIL MOTOR

\section{B. Force Loading System}

Based on the voice-coil motor above, the force loading system of linear oscillating motor can be established. The sketch of the system is depicted in Fig. III. Two linear motors are fixed on the same platform and their output shafts are directly connected via a S-shaped force sensor, which is used to test the load force. The connector on the motor to be tested is bound with a position sensor fixed on the platform for testing the position of the mover. As for control structure, the voicecoil motor is actuated in the force close-loop while the linear oscillating motor is in the position close-loop. Therefore, the voice-coil motor exerts a load force to the other motor according to the velocity of the mover.

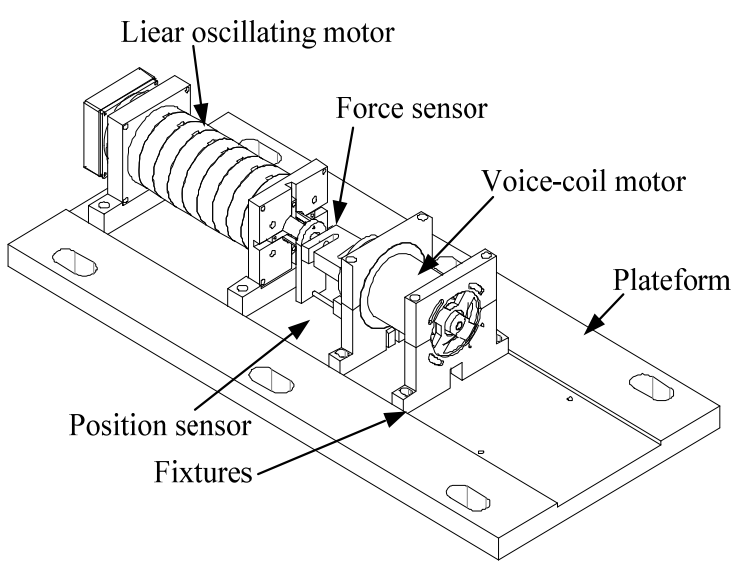

FIGURE III. STRUCTURE OF LINEAR LOADING SYSTEM

\section{ANALYTICAL MODELING OF LOADING Motor}

\section{A. Magnetic Field Density Model}

In this part, the equivalent magnetic circuit method is utilized for the mathematical model of motor in order to obtain the analytical solution of the magnetic density in air gap and the output thrust. In this method, magnetic circuit is analogized to electric circuit and the magnetomotive force is generated by magnets. Besides, the magnetic resistance and flux are analogized to the resistance and the current in electric circuit 
respectively. Before modeling, two assumptions should be made to simplify the actual model.

- The magnetic resistance of back-iron can be ignored due to its large relative permeability.

- The axial component of magnetic density in air gap can be ignored for simplification.

The structure parameters and the magnetic circuit model are shown in Fig. IV and Fig. V respectively.

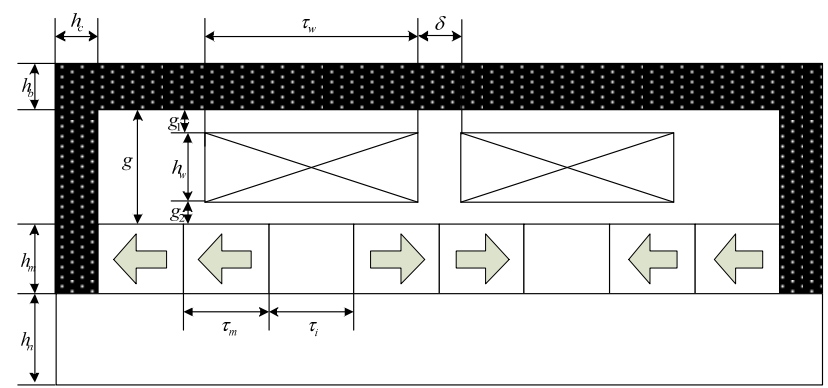

FIGURE IV. STRUCTURE PARAMATERERS OF MOTOR

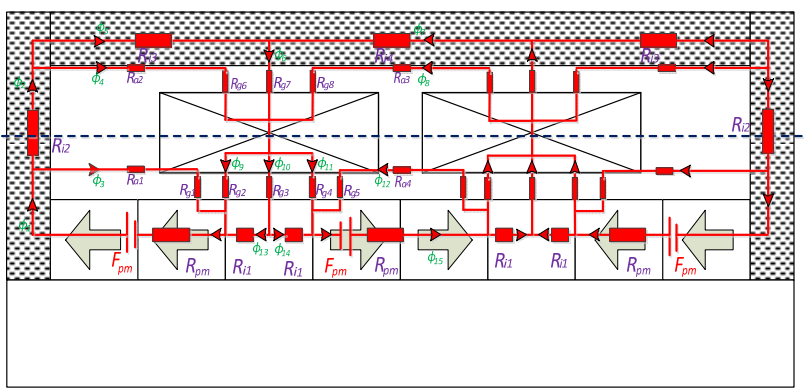

FIGURE V. MAGNETIC CIRCUIT MODEL OF MOTOR

For magnets, the magnetomotive force $F_{p m}$ is given by:

$$
\Phi \pi \mu=\mathrm{H} \chi \cdot 2 \mid \mu .
$$

in which $H_{c}$ represents the coercivity of magnets. According to the definition of the magnetic resistance, which is expressed as:

$$
R_{m}=\frac{l_{m}}{\mu_{0} \mu_{r} \times S_{m}} .
$$

all resistances in magnetic circuit can be achieved, taking $R_{p m}$, $R_{g 1}$ and $R_{a 1}$ as examples:

$$
\begin{gathered}
R_{p m}=\frac{2 \tau_{m}}{\mu_{0} \mu_{r} \times \pi\left[\left(h_{n}+h_{m}\right)^{2}-h_{n}^{2}\right]} . \\
R_{g 1}=\frac{g / 2}{\mu_{0} \times 2 \pi\left(h_{m}+h_{n}+g / 4\right) \times \tau_{m}} . \\
R_{a 1}=\frac{\tau_{m}}{\mu_{0} \times \pi\left[\left(h_{n}+h_{m}+g / 2\right)^{2}-\left(h_{n}+h_{m}\right)^{2}\right]} .
\end{gathered}
$$

with $\mu_{0}$ the permeability of vacuum and $\mu_{r}$ the relative permeability of magnets.

Analogously, Kirchhoff's Law is applied for solving the magnetic flux in each part of the magnetic circuit, which decides the value and the stability of output force. As a result, a fifteen-order group of linear equations can be expressed in the form of matrix:

$$
\left\{\begin{array}{l}
F_{p m}=\phi_{1} \times R_{p m}+\phi_{3} \times\left(R_{a 1}+R_{g 1}\right) \\
F_{p m}=\phi_{15} \times R_{p m}+\phi_{12} \times\left(R_{a 4}+2 R_{g 5}\right) \\
\phi_{2} \times R_{i 2}+\phi_{4} \times\left(R_{a 2}+R_{g 6}\right)+\phi_{9} \times R_{g 2}=\phi_{3} \times\left(R_{a 1}+R_{g 1}\right) \\
\phi_{5} \times R_{i 3}+\phi_{6} \times R_{g 7}=\phi_{4} \times\left(R_{a 2}+R_{g 6}\right) \\
\phi_{13} \times R_{i 1}+\phi_{10} \times R_{g 3}=\phi_{9} \times R_{g 2} \\
\phi_{14} \times R_{i 1}+\phi_{10} \times R_{g 3}=\phi_{11} \times R_{g 4} \\
\phi_{8} \times\left(R_{a 3}+2 R_{g 8}\right)+2 \phi_{11} \times R_{g 4}=\phi_{12} \times\left(R_{a 4}+2 R_{g 5}\right) \\
\phi_{7} \times R_{i 4}+2 \phi_{6} \times R_{g 7}=\phi_{8} \times\left(R_{a 3}+2 R_{g 8}\right) \\
\phi_{1}=\phi_{2}+\phi_{3} \\
\phi_{2}=\phi_{4}+\phi_{5} \\
\phi_{6}=\phi_{5}+\phi_{7} \\
\phi_{4}+\phi_{6}+\phi_{8}=\phi_{9}+\phi_{10}+\phi_{11} \\
\phi_{10}=\phi_{13}+\phi_{14} \\
\phi_{1}=\phi_{3}+\phi_{9}+\phi_{13} \\
\phi_{15}=\phi_{11}+\phi_{12}+\phi_{14}
\end{array}\right.
$$

After solving this system of linear equations, the magnetic field density in the lower air gap is step-shaped, which means it is constant within a certain range in the axial direction and can be derived as:

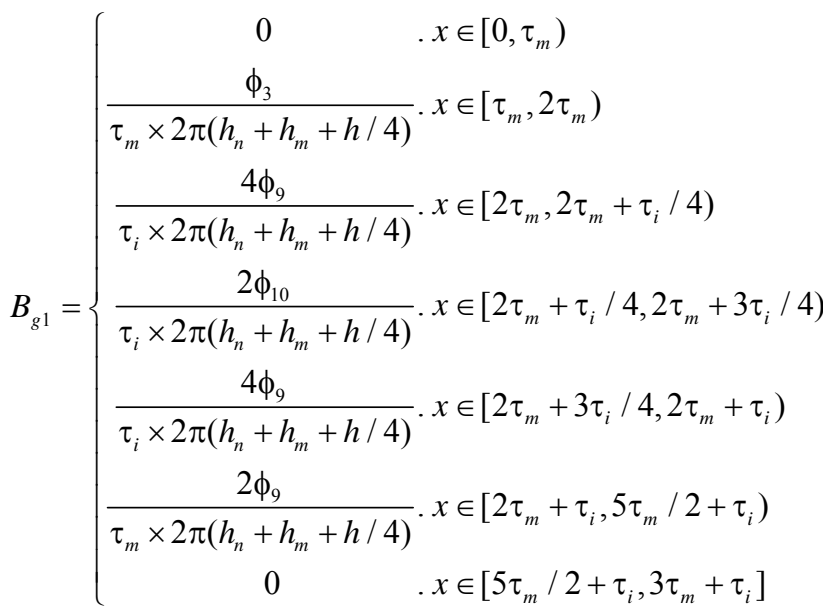

Similarly, the magnetic field density in the upper air gap is given by: 


$$
B_{g 2}=\left\{\begin{array}{cl}
0 & x=0-\tau_{m} \\
\frac{\phi_{4}}{\tau_{m} \times 2 \pi\left(h_{n}+h_{m}+3 h / 4\right)}, & x=\tau_{m}-2 \tau_{m} \\
\frac{\phi_{6}}{\tau_{i} \times 2 \pi\left(h_{n}+h_{m}+3 h / 4\right)}, & x=2 \tau_{m}-\left(2 \tau_{m}+\tau_{i}\right) \\
\frac{2 \phi_{8}}{\tau_{m} \times 2 \pi\left(h_{n}+h_{m}+3 h / 4\right)}, & x=\left(2 \tau_{m}+\tau_{i}\right)-\left(5 \tau_{m} / 2+\tau_{i}\right) \\
0 \quad & x=\left(5 \tau_{m} / 2+\tau_{i}\right)-\left(3 \tau_{m}+\tau_{i}\right)
\end{array} .\right.
$$

\section{B. Output Force Model}

As for the model of output force, according to the magnetic co-energy theory, the magnetic linkage of coils depends on the energizing current $i$ and the position of mover $x$, defined by:

$$
\psi(i, x)=L i+K_{e} x
$$

in which parameters $L$ and $K_{e}$ are the inductance and the output force constant of the motor, respectively. Besides, the energy stored by the magnetic field in air gap is:

$$
W_{c}=\int_{0}^{i} \psi(i, x) \mathrm{d} i=\frac{1}{2} L i^{2}+K_{e} x i
$$

The output force can be expressed as the partial derivate on the position $x$ of magnetic linkage when the current $i$ is constant:

$$
F_{e}=\left.\frac{\partial W_{c}}{\partial x}\right|_{i=c o n s}=\frac{1}{2} i^{2} \frac{\mathrm{d} L(i, x)}{\mathrm{d} x}+K_{e} i=K_{e} i
$$

Where it is assumed that the inductance is independent on the position of mover. Suppose $\Delta x$ is a tiny displacement of the coil within the stroke. The output force constant $K_{e}$ is given by its definition:

$$
K_{e}=\frac{\left.\phi_{w}\right|_{\Delta x}}{\Delta x}=4 \pi N_{c} B_{g}\left(g+h_{n}+h_{m}\right)
$$

in which $N_{c}$ represents the turns of coil. Thus, equation (9) is changed into as follows:

$$
\begin{aligned}
F_{e} & =4 \pi N_{c} B_{g}\left(g+h_{n}+h_{m}\right) \times i \\
& =4 \pi B_{g}\left(g+h_{n}+h_{m}\right) J A_{s} K_{p} .
\end{aligned}
$$

in which $J, A_{s}$ and $K_{p}$ are the current density, the area of coil groove and the spacer factor of motor, respectively and the product of these three variables equals to $N_{c} i$.

\section{SimUlation ANALYSIS OF LOADING Motor}

\section{A. Finite Element Analysis}

Due to the limitation of equivalent magnetic circuit method, the non-linearity of magnets, the saturation of magnetic circuit and the boundary of magnetic field are not taken into consideration. However, the finite element numerical analysis by the software Ansoft Maxwell can help, which verifies the analytical model and guides the optimization of parameters. The Fig. VI shows the distribution cloud of static magnetic field when the mover is located at the midpoint of the stroke.

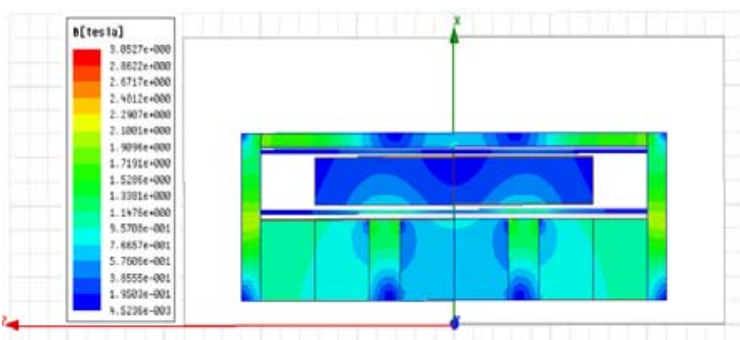

FIGURE VI. DISTRIBUTION CLOUD OF MAGNETIC FIELD

From the distribution cloud above, it is illustrated that the magnetic density at the end cover and the back-iron of the motor are the maximum and doesn't exceed 1.9 T. In addition, compared to the $B-H$ curve of this material, it shows that the magnetic density is located in the linear area and the magnetic circuit is unsaturated, which proves the reasonability of the design for voice-coil motor.

\section{B. Model Verification}

Based on the analysis above, the structural parameters, shown as Tab. I, are utilized for the calculation of the performance parameters of the loading motor.

TABLE I. STRUCTURAL PARAMETERS OF VOICE-COIL MOTOR

\begin{tabular}{|c|c|c|c|}
\hline Item & Symbol & Value & Unit \\
\hline Coil width & $\tau_{w}$ & 36 & $\mathrm{~mm}$ \\
\hline Magnet thickness & $h_{m}$ & 17 & $\mathrm{~mm}$ \\
\hline Upper air gap & $g_{1}$ & 2 & $\mathrm{~mm}$ \\
\hline Lower air gap & $g_{2}$ & 3 & $\mathrm{~mm}$ \\
\hline Coil thickness & $h_{w}$ & 10 & $\mathrm{~mm}$ \\
\hline Magnet width & $\tau_{m}$ & 14 & $\mathrm{~mm}$ \\
\hline Inner iron width & $\tau_{i}$ & 8 & $\mathrm{~mm}$ \\
\hline Diameter of core tube & $h_{n}$ & 10 & $\mathrm{~mm}$ \\
\hline Thickness of outer iron & $h_{b}$ & 3 & $\mathrm{~mm}$ \\
\hline Thickness of end cover & $h_{c}$ & 5 & $\mathrm{~mm}$ \\
\hline
\end{tabular}

Using the analytical model, the radial magnetic flux density in the lower air gap for the proposed voice-coil motor is shown in Fig. VII. The trend in change of the numerical solution (red line) matches well with the analytical one, which provides validity of the analytical model verification. 
Based on this structure, providing rated current for the motor, the output force between analytical model and the numerical solution are compared in Fig. VIII, in which FEM results proves excellent output stability of the motor and good accuracy with acceptable modeling error less than $10 \%$.

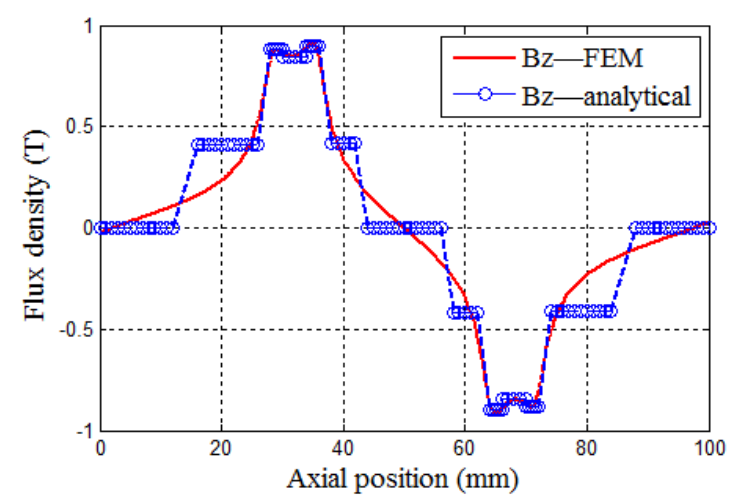

FIGURE VII. COMPARISON BETWEEN ANALYTICAL MODEL AND FEM RESULTS OF FLUX DENSITY IN THE AIR GAP

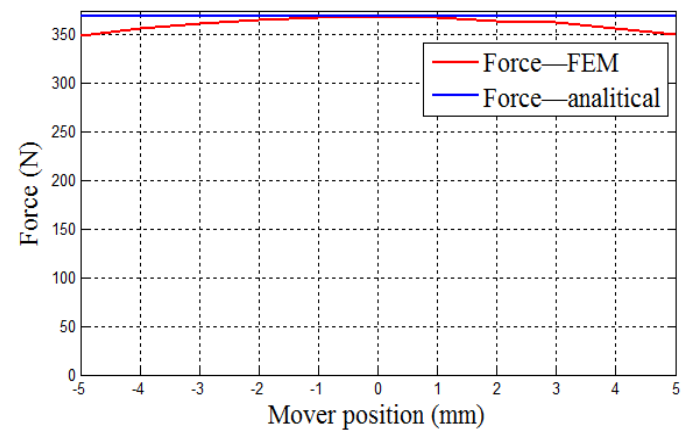

FIGURE VIII. COMPARISON BETWEEN ANALYTICAL MODEL AND FEM RESULTS OF OUTPUT FORCE

\section{CONCLUSION}

This paper presents a loading system for linear oscillating motor based on voice-coil motor because of its low inertia, high frequency response and stable output performance. For the loading motor, analytical modeling using equivalent magnetic circuit method is achieved and compared with the FEM numerical results, proving the reasonability and effectiveness of this design, which provides a feasible solution to high frequency linear loading applications.

\section{ACKNOWLEDGMENT}

The authors acknowledge the National Key Basic Research Program of China (2014CB046406), National Natural Science Foundation of China (NSFC) under grant 51235002, 51575026 and Fundamental Research Funds for the Central Universities.

\section{REFERENCES}

[1] H. Liang, Z. Jiao, L. Yan, L. Zhao, S. Wu and Y. Li, "Design and analysis of tubular linear oscillating motor for directly-driven EHA pump," Sens. Actuator A-Phys., vol. 210, pp. 107-118, 2014. J. Clerk Maxwell, A Treatise on Electricity and Magnetism, 3rd ed., vol. 2. Oxford: Clarendon, 1892, pp.68-73.
[2] J. Wang, Z. Lin and D. Howe, "Analysis of a short-stroke, single-phase, quasi-Halbach magnetised tubular permanent magnet motor for linear compressor applications," IET Electr. Power Appl., vol. 2, no. 3, pp. 193-200, 2008.

[3] J. Wang, W. Wang and K. Atallah, "A linear permanent-magnet motor for active vehicle suspension," IEEE Trans. Veh. Technol., vol. 60, no. 1, pp. 55-63, 2001.

[4] J. Wang, W. Wang, K. Atallah and D. Howe, "Comparative studies of linear permanent magnet motor topologies for active vehicle suspension," IEEE Vehicle Power and Propulsion Conference, pp. 1-6, 2008.

[5] R. Mikalsen and A. P. Roskilly, "A review of free-piston engine history and applications," Appl. Therm. Eng., vol. 27, no. 14-15, pp. 2339-2352, 2007.

[6] Z. Q. Zhu and X. Chen, "Analysis of an E-core interior permanent magnet linear oscillating actuator," IEEE Trans. Magn., vol. 45, no. 10, pp. 4384-4387, 2009.

[7] X. Chen and Z. Q. Zhu, "Analytical determination of optimal split ratio of E-core permanent magnet linear oscillating actuators," IEEE Trans. Ind. Electron., vol. 47, no. 1, pp. 25-33, 2011.

[8] Y. P. Yang and B. J. Huang, "Fuzzy control on the phase and stroke of a linear compressor of a split-stirling cryocooler," Cryogenics, vol. 38, pp. 231-238, 1998.

[9] Y. P. Yang and W. T. Chen, "Dual stroke and phase control and system identification of linear compressor of a split-stirling cryocooler," Asian J. Control, vol. 1, no. 2, pp. 116-121, 1999.

[10] Q. L. Wang, X. Z. Huang, Z. Bo, Q. Tan and J. Li, "The analysis and compensation control of the detent force slot-less tubular permanent magnet linear synchronous motor," IEEE International Conference on Applied Superconductivity and Electromagnetic Devices, pp. 145-146, 2016.

[11] T. Hwang and J. Seok, "Obsever-based ripple force compensation for linear hybrid stepping motor drives," IEEE Trans. Ind. Electron., vol. 54, no. 5, pp. 2417-2424, 2007.

[12] Q. Chen, L. Tao, and Y. Nan, Adaptive nonlinear sliding mode control of mechanical servo system with LuGre friction compensation, vol.138, no. 2, pp. 021003, 2016.

[13] Y. Xia, Q. Lu, Y. Ye and R. Kang, "Modeling and analysis of a novel linear Oscillatory motor with two divided stators," Transactions of China Electrotechnical Society, vol. 22, no. 12, pp. 29-33, 2007.

[14] G. Dong, X. Xu, R. Bai, L. Yu, and X. Qi, Research on electrohydraulic servo loading system, Chinese Hydraulics and Pneumatics, vol.10, pp. 40-45, 2014. 